and select $p \geqq m, p \geqq n$. Let $C=A B+N, D=B A$. Then $L=L C^{p}$ $\oplus \mathfrak{N}\left(C^{p}\right), M=M D^{p} \oplus \mathfrak{N}\left(D^{p}\right)$. We shall prove that $A$ induces an isomorphism $A^{\prime}$ on $L C^{p}$ onto $M D^{p}$. Since for each $j, C^{j} A=A D^{j}$, we have $L C^{p} A=L A D^{p} C M D^{p}$. But $M D^{p}=M D^{p+1}=M B(A B)^{p} A=M B C^{p} A$ $C L C^{p} A$. Thus $A$ is on $L C^{p}$ onto $M D^{p}$. We observe that for any $r$, $C^{r}=(A B)^{r}+(A B)^{r-1} N+\cdots+N^{r}$, hence for $r=2 p, C^{2 p}=(A B)^{2 p}$ $+(A B)^{2 p-1} N+\cdots+(A B)^{p+1} N^{p-1}$ since $N^{p}=N^{m}=0$. If $x C^{p} A=0$, then $x(A B)^{p} A=0, x(A B)^{p+1}=x(A B)^{p+2}=\cdots=0$. Thus $x C^{2 p}=0$, $x C^{p}=0$, which proves that $A^{\prime}$ is an isomorphism. We finally have $C A^{\prime}=A^{\prime} D$ so that the contraction of $C$ to $L C^{p}$ is similar to the contraction of $D$ to $M D^{p}$. Thus $C$ and $D$ have the same elementary divisors which do not have zero as a root. The theorem follows from Theorem 2.

California Institute of Technology

\title{
DIVISION ALGEBRAS OVER FIELDS OF FORMAL POWER SERIES
}

\section{JOHN T. MOORE 1}

1. Introduction. By a field of formal power series we shall mean the field $K$ of all formal power series in $m$ variables $t_{1}, t_{2}, \cdots, t_{m}$ with coefficients in an algebraically closed field of characteristic zero. O. F. G. Schilling has shown that every algebraic extension of finite degree over $K$ is an abelian extension, and the purpose of this note is that of using the result of Schilling to prove the following properties of division algebras over such fields.

Theorem. A central division algebra $D$ over a formal power series field $K$ in $m$ variables is primary if and only if $D$ is cyclic of prime power degree, and the exponent of $D$ is then its degree. Every central division algebra $D$ over $K$ is then a direct product of cyclic algebras.

2. Properties of the coefficient field. We shall be considering a field $K$ which is maximally complete with respect to a valuation function $V$, with values in a discrete linearly ordered abelian group of

Received by the editors January 15, 1951.

1 The results of this paper form a portion of a Ph.D. dissertation, written under the guidance of A. A. Albert. In the remainder of the thesis it is shown that certain division algebras of prime power degree which were constructed by R. Brauer are all primary (Tohôku Math. J. vol. 37 (1933) pp. 77-87). 
rank $m$. Such a field is known ${ }^{2}$ to be isomorphic to the field of formal power series in $m$ variables $t_{1}, t_{2}, \cdots, t_{m}$ with coefficients in a field $F$, which is isomorphic to the field of residue classes. We shall assume, further, that $F$ is algebraically closed of characteristic zero. The elements of $K$ are power series of the form

$$
\sum_{h_{i}=k_{i}}^{\infty} a_{h_{1}, h_{2}}, \cdots, h_{m} t_{1} t_{2}^{h_{1}} t_{2} \cdots t_{m}^{h_{m}}
$$

with rational integral exponents $h_{i}, i=1,2, \cdots, m$, and $a_{h_{1}, h_{2}, \cdots, h_{m}}$ in $F$. The terms of the series are ordered by the lexicographic ordering from the right of the $m$-tuples $\left(h_{1}, h_{2}, \cdots, h_{m}\right)$, and we observe that there are only a finite number of terms with negative exponents. The field $K$ is then the quotient field of the integral domain consisting of all those series whose least term with respect to this ordering is non-negative. The units of $K$ are those elements of the integral domain whose first term is $a_{0,0}, \ldots, 0 \neq 0$. Each element of $K$ can be uniquely expressed in the form $t_{1}^{e_{1}} t_{2}^{e_{2}} \cdots t_{m}^{e_{m}} U$, where $e_{1}, e_{2}, \cdots, e_{m}$ are rational integers and $U$ is a unit. Furthermore, it is known that ${ }^{3}$ all units of $K$ are $n$th powers for any $n$, and that ${ }^{4}$ every algebraic extension of $K$ is abelian and, in fact, a composite of radical fields.

3. Cyclic algebras. Let $D$ be a cyclic division algebra of degree $p^{e}$ over $K$. Since any cyclic field extension of $K$ is a radical extension, and all units of $K$ are $n$th powers for any $n$, we can express $D$ by $\left(Z, S, T_{2}\right)$ where $Z=K\left(T_{1}^{m_{1}}\right), m_{1}=p^{-e}$, and $T_{1}$ and $T_{2}$ are power products of $t_{1}, t_{2}, \cdots, t_{m}$. We can assume that neither $T_{1}$ nor $T_{2}$ is a $p$ th power since the degree of the algebra is $p^{e}$ and it is a division algebra. Suppose that $T_{1}=t_{1}^{d_{1}} t_{2}^{d_{2}} \cdots t_{k}^{d_{k}} \cdots t_{m}^{d_{m}}$, with rational integers $d_{1}, d_{2}, \cdots, d_{k}, \cdots, d_{m}$ which we can assume to be non-negative. The greatest common divisor $d$ of the exponents $d_{1}, d_{2}, \cdots$, $d_{k}, \cdots, d_{m}$ is prime to $p$, if $K\left(T_{1}^{m_{1}}\right)$ has degree $p^{e}$ over $K$. If $\delta=d^{-1}$, this field is also generated by the radical $\left(t_{1}^{d_{1} \delta} t_{2}^{d_{2} \delta} \cdots t_{k}^{d_{k} \delta} \cdots t_{m}^{d_{m} \delta}\right)^{m_{1}}$. At least one exponent $d_{k} \delta=\delta(k)$ is prime to $p$, and so the congruence $\delta(k) x \equiv 1 \bmod p^{e}$ has an integral solution $x$ which is prime to $p$. The field $K\left(T_{1}^{m_{1}}\right)$ is then also generated over $K$ by the radical

$$
\left(t_{1}^{\delta(1) x \delta(2) x} t_{2} \cdots t_{k}^{\delta(k) x} \cdots t_{m}^{\delta(m) x}\right)^{m_{1}}
$$

2 O. F. G. Schilling, Arithmetic in fields of formal power series in several variables, Ann. of Math. vol. 38 (1937) p. 558.

${ }^{3}$ Schilling, loc. cit. p. 561.

4 Schilling, loc. cit. p. 558. 
and so also by

$$
\left(t_{1}^{\delta(1) x t_{2}^{\delta(2) x}} \cdots t_{k} \cdots t_{m}^{\delta(m) x}\right)^{m_{1}} .
$$

Since we can now express $t_{k}=N\left(T_{1}^{m_{1}}\right) \cdot T_{3}^{-1}$, where $N$ represents the norm with respect to $K\left(T_{1}^{m_{1}}\right)$ over $K$ and $T_{3}$ is a power product not involving $t_{k}$, it appears that we can assume that $T_{1}$ contains $t_{k}$ raised to the first power, and $T_{2}$ is a power product not involving $t_{k}$.

It is known ${ }^{5}$ that $D$ has exponent $p^{e}$ if and only if $T_{2}$ is not a norm of the field $K\left(T_{1}^{1 / p}\right)$ over $K$. The multiplicative group of all of the nonzero elements of $K$ which are the norms of elements of $K\left(T_{1}^{1 / p}\right)$ is, ${ }^{6}$ however, the group

$$
\left\{t_{1}^{p}, t_{2}^{p}, \cdots, t_{k-1}^{p}, T_{1}=t_{1}^{\delta(1) x} \cdots t_{k} \cdots t_{m}^{\delta(m) x}, t_{k+1}^{p}, \cdots, t_{m}^{p}\right\} \times \epsilon(K)
$$

where $\epsilon(K)$ is the group of all units of $K$. If $T_{2}$ is a norm, it follows that the equation

$$
T_{2}=t_{1}^{p x_{1}} t_{2}^{p x_{2}} \cdots t_{k-1}^{p x_{k-1}} T_{1}^{x_{k}} t_{k+1}^{p x_{k+1}} \cdots t_{m}^{p x_{m}}
$$

has a nonzero integral solution for $x_{1}, x_{2}, \cdots, x_{k-1}, x_{k}, x_{k+1}, \cdots, x_{m}$. Equating exponents of $t_{k}$, which is not present in $T_{2}$, we must have $x_{k}=0$ and so $T_{2}$ is a $p$ th power. This is a contradiction, and so $T_{2}$ is not a norm of $K\left(T_{1}^{1 / p}\right)$ over $K$. Thus $D$ has exponent $p^{e}$ and we have proved the following theorem.

ThEOREM. The exponent of a cyclic division algebra of degree $p^{e}$ over a field of formal power series $K$ is $p^{e}$.

4. Noncyclic algebras. Let $D$ be any division algebra of degree $p^{\circ}$ over $K$, and let $Z$ be any maximal normal field in $D$. By the results quoted in $\S 2, Z$ is abelian and a composite of radical fields. Thus $Z=K\left(T_{1}^{m_{1}}, T_{2}^{m_{2}}, \cdots, T_{n}^{m_{n}}\right)$ where $T_{1}, T_{2}, \cdots, T_{n}$ are power products of $t_{1}, t_{2}, \cdots, t_{m}$ and $m_{i}=p^{-\theta_{i}}, \sum_{i=1}^{n} e_{i}=e$. Let us further order the above radicals so that

$$
e_{1}=\max \left\{e_{1}, e_{2}, \cdots, e_{n}\right\} .
$$

If we set $L=K\left(T_{2}^{m_{2}}, T_{3}^{m_{3}}, \cdots, T_{n}^{m_{n}}\right)$, we have $Z=L\left(T_{1}^{m_{1}}\right)$ and the $D$-commutator of $L$ is $^{7}$ a central simple algebra of degree $p^{e_{1}}$ over $L$. Since this latter algebra contains the field $L\left(T_{1}^{m_{1}}\right)$, it follows that we

5 A. A. Albert, Structure of algebras, Amer. Math. Soc. Colloquium Publications, vol. 24, p. 98.

6 Schilling, loc: cit. p. 564.

${ }^{7}$ Albert, loc. cit. p. 53. 
can express $D^{L}$ as the cyclic algebra $D^{L}=\left(L\left(T_{1}^{m_{1}}\right), U, \gamma\right)$ for some element $\gamma$ in $L$ and generating automorphism $U$. There exist quantities $X$ and $Y$ in $D^{L}$ such that $X^{p^{\theta_{1}}}=T_{1}$ and $Y^{p_{1}^{e_{1}}}=\gamma$. Since $K(Y)$ is generated by radicals over $K$, it follows that $K(Y)=K\left(\xi_{1}, \xi_{2} \cdots, \xi_{r}\right)$ where $\xi_{i}^{s_{i}}=g_{i}$ in $K, p^{t_{i}}=s_{i}$, with $s_{i}, t_{i}$ positive rational integers. The composite $W=K(Y) \cup L=L(Y)=L\left(\xi_{1}, \xi_{2}, \cdots, \xi_{r}\right)$ is known to be cyclic over $L$ and so must be $L(\xi)$ where $\xi$ is one of the $\xi_{i}$ such that the degree of $L(\xi)$ over $L$ is a maximum. Indeed, every $L\left(\xi, \xi_{j}\right)$ is a subfield of $W$ and is cyclic over $L ; L\left(\xi, \xi_{j}\right)$ is noncyclic unless $\xi_{j}$ is in $L(\xi)$. Then we can take $Y=\xi$ and so have $\gamma^{p^{v}}$ in $K$ for some positive rational integer $v$. Then $Y$ is a radical of index $p^{e_{1}+v}$ over $K$, and $W$ is a splitting field for $D$. If $v=0$, then $\gamma$ is in $K$ and $D^{L}=(K(X), V, \gamma)$ $\times L$ for some automorphism $V$, and so $D=(K(X), V, \gamma) \times D_{1}$ where $D_{1}$ is the $D$-commutator of $(K(X), V, \gamma)$. In this case $D$ is nonprimary, the exponent of $D$ is less than the index of $D$, and $D$ is noncyclic. If $v>0$, we have constructed an abelian splitting field $Z^{\prime}=W=L(\xi)$ with $e_{1}^{\prime}=e_{1}+v>e_{1}$, where $p_{1}^{e_{1}^{\prime}}$ is the maximal index of the radicals over $K$ generating $Z^{\prime}$. This finite process may be continued until it terminates and leads to the conclusion that either $D$ is not primary or that $D$ has a cyclic splitting field. Thus we have the result stated in $\$ 1$. The existence of nonprimary division algebras, over fields of formal power series, with unequal degree and exponent, was established by Schilling ${ }^{8}$ for $m=4$.

University of Chicago

${ }^{8}$ Schilling, loc. cit. p. 575. 